\title{
Response Assessment in Pediatric Neuro-Oncology: Implementation and Expansion of the RANO Criteria in a Randomized Phase II Trial of Pediatric Patients with Newly Diagnosed High-Grade Gliomas
}

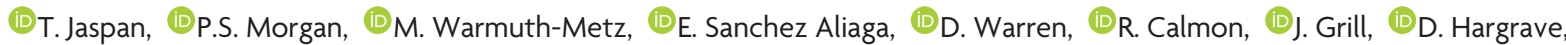
(1). Garcia, and (1) G. Zahlmann

\begin{abstract}
SUMMARY: Determination of tumor response to treatment in neuro-oncology is challenging, particularly when antiangiogenic agents are considered. Nontumoral factors (eg, blood-brain barrier disruption, edema, and necrosis) can alter contrast enhancement independent of true tumor response/progression. Furthermore, gliomas are often infiltrative, with nonenhancing components. In adults, the Response Assessment in Neuro-Oncology (RANO) criteria attempted to address these issues. No such guidelines exist yet for children. The ongoing randomized phase II trial, A Study of Avastin (bevacizumab) in Combination With Temolozomide (TMZ) and Radiotherapy in Paediatric and Adolescent Patients With High-Grade Glioma (HERBY), will establish the efficacy and safety of the antiangiogenic agent bevacizumab for the first-line treatment of newly diagnosed high-grade glioma in children ( $n=121$ patients, enrollment complete). The primary end point is event-free survival (tumor progression/recurrence by central review, second primary malignancy, or death). Determination of progression or response is based on predefined clinical and radiographic criteria, modeled on the RANO criteria and supported by expert pseudoprogression review and the use of standardized imaging protocols. The HERBY trial will also compare conventional MR imaging (T1-weighted and T2/fluid-attenuated inversion recovery sequences) with conventional MR imaging plus diffusion/perfusion imaging for response assessment. It is anticipated that HERBY will provide new insights into antiangiogenic-treated pediatric brain tumors. HERBY will also investigate the practicality of obtaining adequate quality diffusion/perfusion scans in a trial setting, and the feasibility of implementing standard imaging protocols across multiple sites. To date, $61 / 73(83.6 \%)$ patients with available data have completed diffusion-weighted imaging (uptake of other nonconventional techniques has been limited). Harmonization of imaging protocols and techniques may improve the robustness of pediatric neuro-oncology studies and aid future trial comparability.
\end{abstract}

ABBREVIATIONS: HERBY = A Study of Avastin (bevacizumab) in Combination With Temolozomide (TMZ) and Radiotherapy in Paediatric and Adolescent Patients With High-Grade Glioma; HGG = high-grade glioma; RANO = Response Assessment in Neuro-Oncology; RAPNO = Response Assessment in Pediatric Neuro-Oncology

$\mathrm{H}$ igh-grade gliomas (HGGs) are primary malignant tumors of the brain and CNS, which originate from glial cells. HGGs are fast-growing, poorly differentiated, and aggressive. Their location in the brain, their ill-defined borders, and infiltrative nature makes imaging and assessment of response to therapy particularly challenging.

Received November 13, 2015; accepted after revision February 14, 2016. From Nottingham University Hospitals National Health Service Trust (T.J., P.S.M.), Nottingham, UK; Würzburg University (M.W.-M.), Würzburg, Germany; VU University Medical Center (E.S.A.), Amsterdam, the Netherlands; Leeds Teaching Hospital National Health Service Trust (D.W.), Leeds, West Yorkshire, UK; Assistance Publique-Hôpitaux de Paris (R.C.), Paris, France; Gustave Roussy and Paris-Sud University (J.Grill), Villejuif, France; Great Ormond Street Hospital (D.H.), London, UK; and F. Hoffmann-La Roche (J.Garcia, G.Z.), Basel, Switzerland.

This work was sponsored by F. Hoffmann-La Roche. Support for third-party writing assistance for this manuscript was provided by F. Hoffmann-La Roche.

Previously presented in part or whole at: Annual Meeting of the American Society of Clinical Oncology, June 1-5, 2012; Chicago, Illinois; and Annual Meeting of the Society of Neuro-Oncology, November 21-24, 2013; San Francisco, California. American Society of Clinical Oncology trials in progress abstract:

http://meetinglibrary.asco.org/content/94634-114.
Currently, the predominant method for assessment of CNS tumor progression and response is contrast-enhancing conventional T1-weighted MR imaging. ${ }^{1}$ Although widely used, contrast-enhancing conventional T1-weighted MR imaging has limitations in neuro-oncology. The degree of tumor enhancement can be influenced by a variety of neuro-oncology-related factors, including corticosteroid use, imaging technique (1.5T versus 3T, or dose and timing), surgical injury, inflammation, ischemia, edema, infection, seizures, and radiation injury. Increases in contrast enhancement, triggered by nontumoral events, can incorrectly be interpreted as progression (ie, pseudoprogression). Decreases in contrast enhancement, for example by modification of the blood-brain barrier permeability, can be incorrectly inter-

Please address correspondence to Tim Jaspan, MD, Imaging Centre, B Floor, Nottingham University Hospitals National Health Service Trust, Nottingham, NG7 2UH, UK; e-mail: tjaspan@doctors.org.uk

三 Indicates article with supplemental on-line tables.

$\Delta$ Indicates article with supplemental on-line photos.

http://dx.doi.org/10.3174/ajnr.A4782

AJNR Am J Neuroradiol 37:1581-87 Sep 2016 www.ajnr.org 
preted as response (ie, pseudoresponse). In adults with newly diagnosed glioblastomas treated with radiation therapy and temozolomide (the current standard of care), rates of pseudoprogression of $21 \%-31 \%$ have been reported. ${ }^{2,3}$ The increasing interest in antiangiogenic therapies for highly vascularized brain tumors further compounds these concerns; antiangiogenic treatment can improve blood-brain barrier function, decrease enhancement (pseudoresponse), cause transient normalization of tumor vasculature, and may mask tumor changes. Finally, some tumor growth can be non-contrast-enhancing and not detected by conventional MR imaging.

Guidelines for tumor assessment in neuro-oncology were updated in 2010 by the Response Assessment in Neuro-Oncology (RANO) working group (On-line Table 1). ${ }^{4,5}$ These guidelines, based on evaluations of adult HGGs, replaced the previously used Macdonald criteria and aimed to provide more specific and standardized imaging protocols. The RANO working group recommended the use of T2 and FLAIR sequences to assess nonenhancing tumor growth. The potential for pseudoprogression was partially addressed but fell short of definitively distinguishing pseudoprogression from true progression; within the first 12 weeks of completion of radiation therapy, progression can only be determined if most of the new enhancement is outside of the radiation field (as per RANO criteria, an example would be enhancement beyond the high-dose region or $80 \%$ isodose line) or if there is histologic confirmation following further resection. The phase III, placebo-controlled AVAglio trial (A Study of Avastin [Bevacizumab] in Combination With Temozolomide and Radiotherapy in Patients With Newly Diagnosed Glioblastoma) for adults with newly diagnosed glioblastoma, which investigated the addition of bevacizumab to radiation therapy plus temozolomide, incorporated aspects of the RANO criteria (though it was initiated before these criteria were published). ${ }^{6}$ The AVAglio protocol prespecified methods to identify pseudoprogression in a consistent manner and used T2/FLAIR sequences to assess nonenhancing lesions. ${ }^{7}$ Progression-free survival benefits were reported with the addition of bevacizumab to standard-of-care treatment in newly diagnosed glioblastoma. To date, there have been no such studies in pediatric patient populations, and extrapolation of results from adults to children is not appropriate. Furthermore, it is not known whether the RANO criteria could be directly implemented into pediatric populations without modification.

\section{Response Assessment in Pediatric Neuro-Oncology}

The assessment of response and progression in pediatric brain tumors brings further challenges, such as the brain still being under development in childhood. Pediatric brain tumors tend to be heterogeneous with varying histology. Glial tumors, in particular, have diverse morphology and variable blood-brain barrier integrity and may be poorly circumscribed. ${ }^{7}$ Due to the variation in tumors within the pediatric population and the relative rarity of each individual tumor type, it can be difficult for individual study centers to gain experience in imaging each type.

To date, most neuro-oncology research and development has focused on adult trials, and randomized clinical trials in pediatric patients are few. Accordingly, no clear relationship between tumor response and improved survival has been established in pediatric patients. Where clinical studies have been conducted, the populations tend to include a wide age range. Due to the limited information available there are, as yet, no agreed standards to define response and progression in children. Thus, the adult RANO criteria may not be directly applicable to pediatric brain tumors. A working group is examining this issue (Response Assessment in Pediatric Neuro-Oncology [RAPNO]). ${ }^{7}$ Given the challenges associated with pediatric brain tumors, several diagnostic uncertainties remain, including the following: the accuracy of metrics for assessing tumor response, the best way to measure enhancing tumors, whether all enhancement can be attributed to tumor tissue, whether loss of enhancement is a surrogate for response, the best way to assess nonenhancing tumors, and understanding how imaging changes correlate with clinical and neurologic status.

\section{The HERBY Trial}

A clinical trial was set up to establish the efficacy and safety of the antiangiogenic agent bevacizumab for the first-line treatment of newly diagnosed HGG in children (A Study of Avastin [bevacizumab] in Combination With Temolozomide [TMZ] and Radiotherapy in Paediatric and Adolescent Patients With High-Grade Glioma [HERBY], NCT01390948). The study has been uniquely designed to optimize the assessment of response to treatment to ensure confidence in the data by randomizing the new treatment allocation on top of the standard of care and to inform future work in this patient population with an imaging and biologic repository. In HERBY, the determination of progression or recurrence was mandated on the basis of meeting predefined clinical and radiographic criteria. In addition to investigating bevacizumab treatment, the HERBY study was required to compare the utility of "structural imaging" versus "structural plus multimodal imaging” (Fig 1).

\section{Structural and Multimodal Imaging}

Structural imaging includes standard (conventional) MR imaging (as per the RANO criteria; ie, for assessing T1-enhancing lesions [On-line Fig $1 A$ ] and using FLAIR and T2 sequences for nonenhancing lesions). However, conventional MR imaging provides limited information on physiologic tumor characteristics and the status of the perilesional tissue. Multimodal imaging uses a variety of techniques to generate physiologic and biochemical information to complement structural imaging. ${ }^{8}$ For example, DWI or DTI with ADC mapping can be used to evaluate cellular density and tissue injury (On-line Figs $1 B$ and $2 C$ ). Dynamic susceptibility contrast (perfusion) MR imaging can provide an indication of tumor vascularity and capillary permeability (On-line Figs $1 D$ and $2 E) .{ }^{1} \mathrm{H}$-MR spectroscopy is used to detect metabolites reflecting tissue integrity, disruption, and necrosis (eg, aromatic amino acids, choline, lipids). These advanced imaging techniques are effective in the evaluation of tumor behavior and have been used as surrogate markers of response to tumor-modulating therapy. ${ }^{9-17}$ Furthermore, diffusion and perfusion techniques have been used to identify radiation necrosis ${ }^{9}$ and pseudoprogression. ${ }^{10}$ 


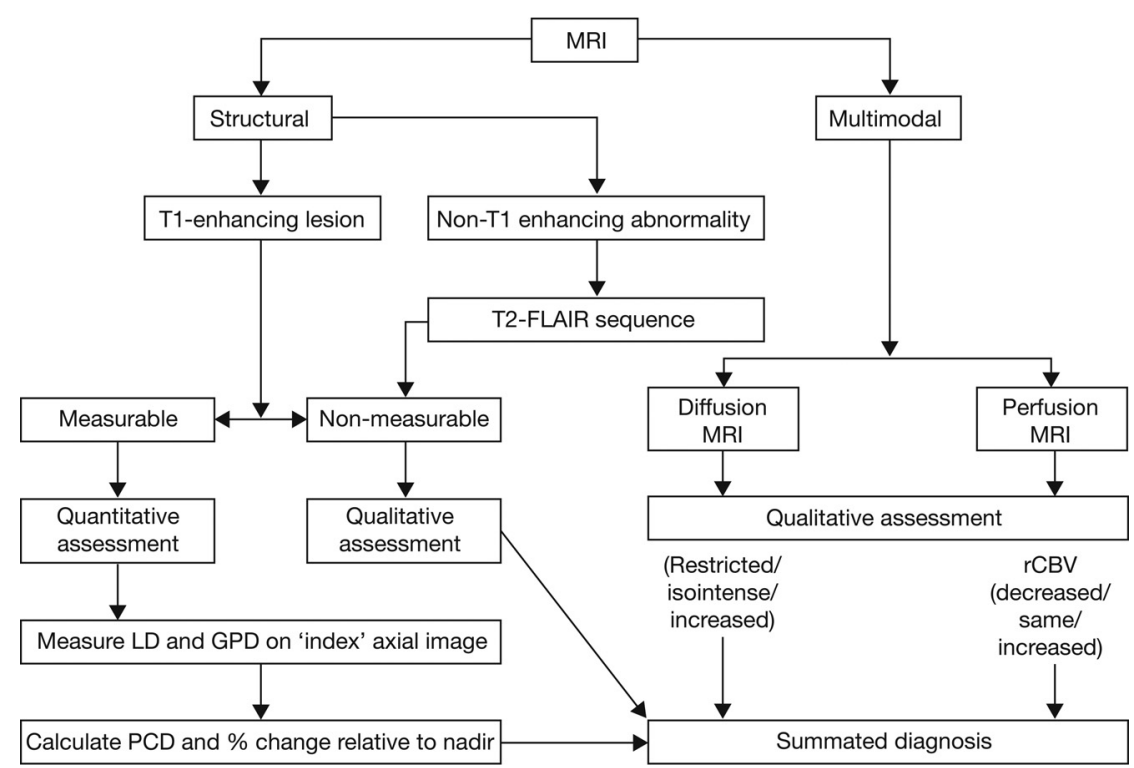

FIG 1. Structural and multimodal imaging used in the HERBY study. LD indicates longest diameter; GPD, greatest perpendicular diameter; PCD, product of cross-sectional enhancing diameters.

Compared with structural MR imaging alone, a combination of structural MR imaging and multimodal imaging represents a greater biologic approach to imaging and provides more of the "big picture" of tumor response. When used in isolation, the additional techniques described are of limited utility, but in combination with structural imaging and clinical assessment, multimodal imaging should provide an enhanced ability to assess tumor grade, viability, and response to therapy.

Implementation of multimodal imaging tools into a clinical trial requires careful consideration. To date, the techniques have been largely experimental and not all technologies are available at all clinics. Standard protocols have not yet been established. ${ }^{1} \mathrm{H}-\mathrm{MR}$ spectroscopy, in particular, is still very exploratory and difficult to standardize. Diffusion and perfusion imaging are the most accessible and available techniques, but there are specific difficulties in undertaking perfusion imaging in the pediatric population due to the size of the patient (eg, obtaining adequate venous access). Regardless of imaging techniques, variability also poses a challenge for the assessment of tumor progression and response; there is intrinsic interobserver variability in the assessment of MR imaging scans, and response to therapy within a tumor itself may be variable. It is also not possible to separate treatment effects from disease effects. As part of the HERBY trial, our aims were the following: 1) to evaluate the implementation of the RANO criteria in a phase II trial of pediatric patients with HGG, 2) to assess the practicality of obtaining multimodal imaging of adequate quality, and 3) to assess the feasibility of including diffusion and perfusion into the response criteria.

\section{MATERIALS AND METHODS \\ Study Design and Patients}

The overall objectives of the HERBY trial are to evaluate the efficacy, safety, tolerability, and pharmacokinetics of bevacizumab when added to postoperative radiation therapy with concomitant and adjuvant temozolomide and to determine whether the addition of bevacizumab to the management of newly diagnosed HGG in children confers a clinical benefit. It was planned to recruit 120 patients during 36 months. Enrollment is complete (121 patients have been enrolled), and the trial is ongoing as of June 2015.

As with the adult AVAglio trial, the pediatric HERBY trial is investigating the addition of bevacizumab to radiation therapy plus temozolomide in a randomized study design, but there are important differences; for example, unlike AVAglio, HERBY allowed inclusion of grade III and grade IV gliomas, was not placebo-controlled, and had no bevacizumab monotherapy component.

Patients (between 3 and 18 years of age) with histologically confirmed, localized supratentorial/infratentorial cerebellar or peduncular (non-brain stem) HGG, adequate organ function, and available preoperative MR imaging were enrolled. Written, informed consent was obtained from the patient, parent, or legal representative, after institutional review board/ethics committee approval was obtained in each recruiting country. After the operation, patients underwent a postoperative MR imaging and were randomized to receive 1 of the following 2 regimens: standard arm, radiation therapy (1.8 Gy/day) and temozolomide $\left(75 \mathrm{mg} / \mathrm{m}^{2} /\right.$ day $)$ for 6 weeks, followed by a 4 -week treatment break, then up to 12 fourweek cycles of temozolomide $\left(150-200 \mathrm{mg} / \mathrm{m}^{2} /\right.$ day); or experimental arm, bevacizumab ( $10 \mathrm{mg} / \mathrm{kg}$ every 2 weeks $)$ plus radiation therapy ( $1.8 \mathrm{~Gy} /$ day) and temozolomide $\left(75 \mathrm{mg} / \mathrm{m}^{2} /\right.$ day) for 6 weeks, followed by bevacizumab during a 4 -week temozolomide break, and then bevacizumab ( $10 \mathrm{mg} / \mathrm{kg}$ every 2 weeks) concomitantly with up to 12 four-week cycles of adjuvant temozolomide (150-200 mg/m²/day) (On-line Fig 2). Patients will be randomized centrally by an interactive voice response system.

The primary end point is event-free survival, defined as the earliest occurrence of tumor progression/tumor recurrence (determined by a Central Radiology Review Committee), second primary non-HGG malignancy, or death. Secondary end points include the following: investigator-assessed event-free survival; overall survival and 1-year overall survival rate; 6-month and 1-year event-free survival rate; overall response rate determined by the Central Radiology Review Committee using RANO criteria; tumor functional changes on diffusion/ perfusion MR imaging for correlative analysis in addition to structural imaging and efficacy outcome measurements; health status; neuropsychologic function; safety; feasibility; and tolerability.

HERBY has an Independent Data Monitoring Committee. This committee shares the responsibility for regularly monitoring the overall safety of patients in the trial with the sponsor and the Trial Steering Group. By carefully reviewing overall death rates, serious adverse events, and other specified safety events by treatment arms, the Independent Data Monitoring Committee provides the sponsor and the Trial Steering Group 
their independent recommendations and helps to minimize patient exposure to unnecessary risk. The Independent Data Monitoring Committee will also evaluate benefits and risks by reviewing the efficacy, safety, and tolerability data during the scheduled futility analysis.

\section{Radiographic and Clinical Response Assessment}

Response evaluations will be performed by using RANO criteria by blinded site-independent central review; radiologic and clinical evaluations will be combined to provide an integrated disease assessment (Fig 2). Efficacy assessments will be performed after the chemoradiotherapy phase and treatment break but before the start of adjuvant temozolomide treatment, and thereafter every 3 months.

For radiographic response, both enhancing (T1-weighted) and nonenhancing lesions (T2-FLAIR sequences) will be considered (Fig 2) according to prespecified criteria for progression (Table). A detailed standardized imaging protocol has been provided to site investigators (On-line Table 2). Both measurable and nonmeasurable disease will be considered. Measurable disease has been defined as contrast-enhancing lesions with clearly defined

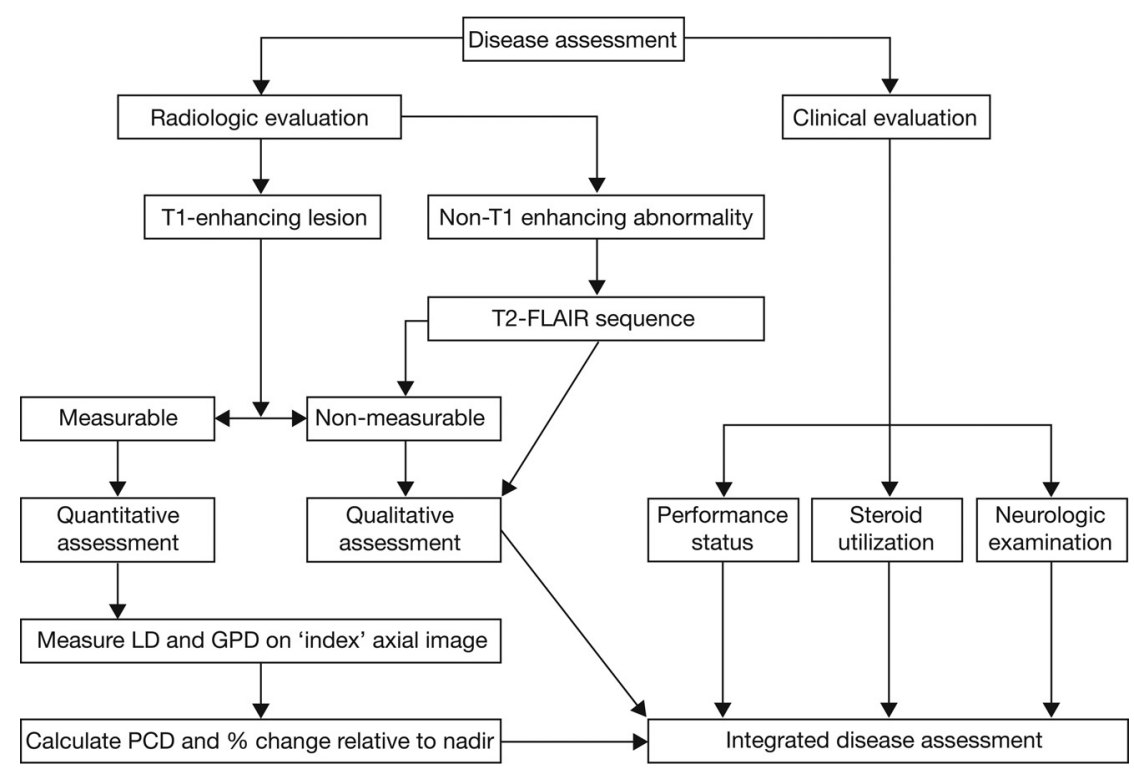

FIG 2. Response evaluation assessment algorithm. LD indicates longest diameter; GPD, greatest perpendicular diameter; PCD, product of cross-sectional enhancing diameters. margins on T1-weighted MR imaging with 2 perpendicular diameters of $\geq 10 \mathrm{~mm}$. Nonmeasurable enhancing tumors have been defined as unidimensional measurable lesions, lesions with margins not clearly defined, or lesions with at least 1 diameter of $<10 \mathrm{~mm}$.

The definition of clinical deterioration has been left to the discretion of the treating physician, but it was recommended that substantial decreases in the Karnofsky Performance or Lansky Play Scales be considered deterioration. Increases in corticosteroid doses will also contribute to the evaluation of clinical deterioration.

\section{Pseudoprogression}

The risk of pseudoprogression is highest within 12 weeks of chemoradiotherapy completion; during this period in the HERBY trial, clinical decline alone will be considered insufficient to determine disease progression. Progression will be determined only if there is new enhancing disease that extends outside the radiation field or if there is unequivocal evidence of viable tumor on histology (On-line Fig 3). Experienced pediatric neuroradiologists will be available during the trial to assist local radiologists in the evaluation of progression during the early postchemoradiation period. The expert reviewer will provide an opinion to distinguish pseudoprogression from true progression, but the site investigator will make the final decision. Imaging evaluations will be performed again at the next visit (beyond the 12week period), and if there is a continued increase in enhancing lesions, then the first scan will be considered to have represented true progression. If the lesion is decreased or stable compared with the first posttreatment scan, the increase at the preceding time point will be considered pseudoprogression.

\section{Multimodal Imaging}

Where participating centers have access to multimodal imaging, an appended protocol will be performed in addition to standard MR imaging. Guideline imaging parameters have been provided in the HERBY protocol for DWI and dy-

Criteria for tumor progression in HERBY (adapted from the RANO criteria)

\begin{tabular}{|c|c|}
\hline Parameter & Criterion \\
\hline $\begin{array}{l}\text { T1-weighted post-Gd } \\
\text { measurable disease }\end{array}$ & $\begin{array}{l}\geq 25 \% \text { increase in the sum of the products of perpendicular diameters of the contrast-enhancing lesions } \\
\text { compared with the smallest tumor measurement obtained either at baseline (if no decrease was observed) } \\
\text { or best response with the patient on stable or increasing doses of corticosteroids }\end{array}$ \\
\hline $\begin{array}{l}\text { T1-weighted post-Gd } \\
\text { nonmeasurable disease }\end{array}$ & Clear progression of nonmeasurable lesions \\
\hline \multirow[t]{2}{*}{ T2/FLAIR } & $\begin{array}{l}\text { Substantial increase in the T2/FLAIR nonenhancing lesion compared with the baseline scan or best response } \\
\text { after the initiation of therapy with the patient on a stable or increasing dose of corticosteroids }\end{array}$ \\
\hline & $\begin{array}{l}\text { The increase in the T2/FLAIR signal must have occurred and not be the result of comorbid events (eg, radiation } \\
\text { therapy, demyelination, ischemic injury, seizures, postoperative changes, or other treatment effects) }\end{array}$ \\
\hline New lesions & Any new lesions \\
\hline Clinical & $\begin{array}{l}\text { Clear clinical deterioration due to tumor and not attributable to other causes (eg, seizures, medication } \\
\text { adverse effects, complications of therapy, cerebrovascular events, infection, and so forth) }\end{array}$ \\
\hline Failure to return & Failure to return for evaluation as a result of death or deteriorating condition \\
\hline
\end{tabular}

Note:-Gd indicates gadolinium. 
namic susceptibility contrast imaging (On-line Table 3). A correlative analysis of functional changes in tumor on MR diffusion/ perfusion imaging and efficacy outcome measures is to be performed at the time of the primary analysis. Correlative studies of ${ }^{1} \mathrm{H}-\mathrm{MR}$ spectroscopy were planned for HERBY, but only a small number of ${ }^{1} \mathrm{H}$-MR spectroscopy scans have been acquired to date, which will likely prove to be insufficient for analysis. Decision rules for assigning response status are presented in On-line Table 4. For example, if structural MR imaging indicated "complete response," but diffusion status was decreased and perfusion status was "normal/decreased," the combined status will be revised to "stable disease."

\section{Statistical Analysis Plan}

For event-free survival and overall survival, Kaplan-Meier curves will be generated and 95\% confidence intervals will be estimated by using the Rothman method. An estimate of the treatment effect will be expressed as the hazard ratio with corresponding 95\% CI (estimated in a Cox regression analysis). Analyses comparing treatment arms will include the 2-sided log-rank test at the $5 \% \alpha$ level. Event-free survival and overall survival rates will be estimated by using the Kaplan-Meier method with 95\% CIs calculated by using the Greenwood formula. The difference in overall response rate between the treatment arms will be tested in an exploratory manner, by using a $\chi^{2}$ test with Schouten correction, with 95\% Pearson-Clopper CIs. We will calculate 95\% HauckAnderson CIs for the difference in response rates between the treatment arms. Other analyses will be descriptive.

\section{Current Status of HERBY}

At the time of manuscript preparation, recruitment into the HERBY trial has been completed $(n=121)$ and the study is ongoing. To date, the RANO criteria have been successfully applied (by using the central radiologic read) for the first 73 patients.

\section{DISCUSSION}

The HERBY study aims, primarily, to establish whether bevacizumab, when added to radiation therapy and temozolomide, can improve clinical outcomes for pediatric patients with HGG. However, an additional aim of HERBY is to assess the value of the structural imaging (based on the RANO criteria) alone and when combined with multimodal imaging for pediatric neuro-oncology trials, with the potential of guiding future recommendations (RAPNO). Accordingly, the RANO criteria have been implemented into the HERBY trial, with some modifications. For example, the use of an expert reviewer for identification of pseudoprogression was incorporated into the study design. In addition, to reduce bias and variability, site-independent central review will be used to determine response to treatment.

Tumor diffusion and perfusion imaging have also been incorporated to complement the RANO criteria, to investigate whether these tools can enhance the understanding of tumor response to bevacizumab in pediatric patients with HGG. To date, the use of these tools has been variable in HERBY. Although most sites claimed that they were able to do full multimodal imaging before the trial started, the uptake has been poor for some techniques. DWI was the most prevalent technique used; its value in the context of the RANO criteria is still to be evaluated, but currently it seems that DWI is the most likely to be pragmatically incorporated into the revised RANO criteria for pediatric patients.

A key aspect of HERBY is the provision of detailed protocols for the imaging procedures, which aim to help standardize assessment across sites and countries. Standardized imaging protocols are the subject of recent draft FDA guidance, which recommends that trials augment existing standards to create trial-specific imaging standards, particularly in cases in which images can be interpreted quantitatively. ${ }^{18}$ The guidance also recommends that when imaging modalities are vulnerable to image-quality problems, central imaging interpretation should be considered.

Standardization is important for both conventional MR imaging and the diffusion/perfusion techniques being used in HERBY, these latter being, to date, largely experimental. Future trials will require a consensus on protocols with consistent implementation to maximize their value. To this end, following a workshop held in January 2014, a recent consensus publication has outlined a standardized brain tumor anatomic imaging protocol, involving the following: patient advocates; device and pharmaceutical industry leaders; the FDA; the National Cancer Institute; and academic experts in neuro-oncology, neuroradiology, and imaging physics. ${ }^{19}$ The recommended protocol reflects recent imaging developments, particularly the use of 3D isotropic T1-weighted imaging pre- and post-gadolinium administration to enable highquality T1-subtraction image acquisition, a technique that potentially offers improved tumor visualization and quantification of enhancing tumoral tissue. ${ }^{20}$ The proposed protocol (On-line Table 5) builds on and refines that proposed within the RANO publications and reflects the dynamic and changing status of neurooncology imaging. While T1-subtraction images will be evaluated in HERBY, for the most part, this evaluation will depend on 2D T1-weighted image acquisitions; the protocol for the study was derived in 2010 before the current recommendations for T1 volume imaging.

The HERBY study design has several potential strengths, which are anticipated to reinforce the results. For example, the use of central review and decision rules should bring consistency to response assessment and reduce variability. HERBY also includes algorithms for early progression (potential pseudoprogression), which have proved valuable in adult trials ${ }^{6}$; and it incorporates detailed imaging protocols, which could potentially be used as a standard in future pediatric HGG imaging. HERBY has a relatively large patient number in comparison with other pediatric trials; however, there may be variability within the 121 patients that precludes clear conclusions on all aspects under investigation.

We hope that HERBY will meet its aims of establishing the efficacy and safety of a new regimen for the first-line treatment of newly diagnosed high-grade glioma, a condition for which new, effective treatments are very much needed. However, regardless of outcomes, it is anticipated that HERBY will provide valuable new insights into pediatric brain tumors treated with antiangiogenic therapy, which can be applied to future work. HERBY is the first study to compare standard structural imaging with structural im- 
aging plus diffusion/perfusion imaging and will give an indication of whether the latter influences the perception of tumor response or progression. This randomized trial will provide valuable information on the true clinical value of these additional imaging tools. The availability of DWI and structural imaging should allow some conclusions to be drawn on the value of this technique for pediatric HGG response assessment in clinical practice. Furthermore, HERBY will allow assessment of the feasibility and practicality of using standardized imaging protocols in clinical trials.

\section{CONCLUSIONS}

RANO criteria can be implemented into pediatric trial protocols, but this requires careful consideration; we implemented a review of potential pseudoprogression by expert readers and validation of radiographic progression in the case of stable clinical parameters. The HERBY trial will assess, for the first time, whether functional imaging techniques such as diffusion and perfusion MR imaging, can strengthen the assessment of response when combined with conventional imaging tools. HERBY has also introduced standardized imaging protocols and augmented the RANO criteria with central review and expert advisors. Harmonization of imaging protocols and techniques may improve the robustness of pediatric neuro-oncology studies and aid future trial comparability.

\section{ACKNOWLEDGMENTS}

We thank the patients and parents who are participating in the HERBY study and the staff at the study sites; Louis Viviers (Institut Gustave Roussy) for initial generation of tables and figures; and Dawn Saunders, Lee Coleman, and Lisbeth Reneman for their contributions. We thank Dr Gilles Vassal for his review of the manuscript. The HERBY central review radiology reviewers are Tim Jaspan, Monika Warmuth-Metz, Esther Sanchez Aliaga, Daniel Warren, and Raphael Calmon. The HERBY Trial Steering Group includes Tim Jaspan, Paul S. Morgan, Jacques Grill, and Darren Hargrave. The HERBY Radiology Committee comprises Tim Jaspan, Paul S. Morgan, Monika Warmuth-Metz, Esther Sanchez Aliaga, Daniel Warren, Raphael Calmon, and Gudrun Zahlmann.

Disclosures: Timothy Jaspan—RELATED: Grant: Instituit Gustav Roussy, which, in turn, received funding from Roche, ${ }^{*}$ Comments: grant to undertake primary and secondary endpoint radiologic imaging analysis; Support for Travel to Meetings for the Study or Other Purposes: Instituit Gustav Roussy, which, in turn, received funding from Roche, ${ }^{*}$ Comments: attendance at European Society of Paediatric Oncology and International Symposium on Pediatric Neuro-Oncology meetings in 2014 and 2015; Fees for Participation in Review Activities such as Data Monitoring Boards, Statistical Analysis, Endpoint Committees, and the Like: Instituit Gustav Roussy, which, in turn, received funding from Roche, ${ }^{\star}$ Comments: attendance at Trial Steering Group meetings. Paul S. Morgan—RELATED: Grant: Roche, ${ }^{\star}$ Comments: support for assistance for MR imaging aspects of the HERBY study; Support for Travel to Meetings for the Study or Other Purposes: Roche, ${ }^{\star}$ Comments: support for travel to meetings related to the HERBY study; Fees for Participation in Review Activities such as Data Monitoring Boards, Statistical Analysis, Endpoint Committees, and the Like: Roche, ${ }^{*}$ Comments: for attendance at the Trial Steering Group of the HERBY study. Monika Warmuth-Metz-RELATED: Consulting Fee or Honorarium: Roche, Comments: payment for blinding evaluation of images; Support for Travel to Meetings for the Study or Other Purposes: Roche, Comments: payment of travel expenses for the imaging review training; UNRELATED: Grants/Grants Pending: German Children's Cancer Foundation, ${ }^{*}$ Comments: money for the evaluation of imaging of all patients in the German brain tumor study. The German Children's Cancer Foundation is a parents' association, and the money we receive is provided by donations. Esther Sanchez Aliaga—RELATED: Consulting Fee or Honorarium: F. Hoffmann-La
Roche; Support for Travel to Meetings for the Study or Other Purposes: F. Hoffmann-La Roche. Daniel Warren-RELATED: Support for Travel to Meetings for the Study or Other Purposes: F. Hoffmann-La Roche; Fees for Participation in Review Activities such as Data Monitoring Boards, Statistical Analysis, Endpoint Committees, and the Like: F. Hoffmann-La Roche; UNRELATED: Consultancy: F. Hoffmann-La Roche. Raphael Calmon—RELATED: Support for Travel to Meetings for the Study or Other Purposes: F. Hoffmann-La Roche; Fees for Participation in Review Activities such as Data Monitoring Boards, Statistical Analysis, Endpoint Committees, and the Like: F. Hoffmann-La Roche. Jacques Grill—UNRELATED: Grants/Grants Pending: Novartis, ${ }^{*}$ Roche, ${ }^{*}$ Bristol-Myers Squibb. ${ }^{*}$ Darren Hargrave-RELATED: Support for Travel to Meetings for the Study or Other Purposes: Roche, Comments: expenses paid for Trial Steering Group meeting; UNRELATED: Consultancy: I have provided consultancy for Roche for bevacizumab plus other portfolio products. Josep Garcia-OTHER RELATIONSHIPS: I am a clinical scientist working at Roche, which is disclosed already as part of my institution. Gudrun Zahlmann—UNRELATED: Employment: F. Hoffmann-La Roche; Stock/Stock Options: employee program of the company. *Money paid to the institution.

\section{REFERENCES}

1. Dhermain FG, Hau P, Lanfermann H, et al. Advanced MRI and PET imaging for assessment of treatment response in patients with gliomas. Lancet Neurol 2010;9:906-20 CrossRef Medline

2. Brandes AA, Franceschi E, Tosoni A, et al. MGMT promoter methylation status can predict the incidence and outcome of pseudoprogression after concomitant radiochemotherapy in newly diagnosed glioblastoma patients. J Clin Oncol 2008;26:2192-97 CrossRef Medline

3. Taal W, Brandsma D, de Bruin HG, et al. Incidence of early pseudoprogression in a cohort of malignant glioma patients treated with chemoirradiation with temozolomide. Cancer 2008;113:405-10 CrossRef Medline

4. Wen PY, Macdonald DR, Reardon DA, et al. Updated response assessment criteria for high-grade gliomas: response assessment in neuro-oncology working group. J Clin Oncol 2010;28:1963-72 CrossRef Medline

5. Chinot OL, Macdonald DR, Abrey LE, et al. Response assessment criteria for glioblastoma: practical adaptation and implementation in clinical trials of antiangiogenic therapy. Curr Neurol Neurosci Rep 2013;13:347 CrossRef Medline

6. Chinot OL, Wick W, Mason W, et al. Bevacizumab plus radiotherapy-temozolomide for newly diagnosed glioblastoma. $N$ Engl J Med 2014;370:709-22 CrossRef Medline

7. Warren KE, Poussaint TY, Vezina G, et al. Challenges with defining response to antitumor agents in pediatric neuro-oncology: a report from the response assessment in pediatric neuro-oncology (RAPNO) working group. Pediatr Blood Cancer 2013;60:1397-401 CrossRef Medline

8. Kang TW, Kim ST, Byun HS, et al. Morphological and functional MRI, MRS, perfusion and diffusion changes after radiosurgery of brain metastasis. Eur J Radiol 2009;72:370-80 CrossRef Medline

9. Cha J, Kim ST, Kim HJ, et al. Analysis of the layering pattern of the apparent diffusion coefficient (ADC) for differentiation of radiation necrosis from tumour progression. Eur Radiol 2013;23:879-86 CrossRef Medline

10. Lee WJ, Choi SH, Park CK, et al. Diffusion-weighted MR imaging for the differentiation of true progression from pseudoprogression following concomitant radiotherapy with temozolomide in patients with newly diagnosed high-grade gliomas. Acad Radiol 2012;19: 1353-61 CrossRef Medline

11. Chang YW, Yoon HK, Shin HJ, et al. MR imaging of glioblastoma in children: usefulness of diffusion/perfusion-weighted MRI and MR spectroscopy. Pediatr Radiol 2003;33:836-42 CrossRef Medline

12. Batchelor TT, Gerstner ER, Emblem KE, et al. Improved tumor oxygenation and survival in glioblastoma patients who show increased blood perfusion after cediranib and chemoradiation. Proc Natl Acad Sci U S A 2013;110:19059-64 CrossRef Medline

13. Nasseri M, Gahramanov S, Netto JP, et al. Evaluation of pseudoprogression in patients with glioblastoma multiforme using dynamic magnetic resonance imaging with ferumoxytol calls 
RANO criteria into question. Neuro Oncol 2014;16:1146-54 CrossRef Medline

14. Bag AK, Cezayirli PC, Davenport JJ, et al. Survival analysis in patients with newly diagnosed primary glioblastoma multiforme using pre- and post-treatment peritumoral perfusion imaging parameters. J Neurooncol 2014;120:361-70 CrossRef Medline

15. Ratai EM, Zhang Z, Snyder BS, et al. Magnetic resonance spectroscopy as an early indicator of response to anti-angiogenic therapy in patients with recurrent glioblastoma: RTOG 0625/ACRIN 6677. Neuro Oncol 2013;15:936-44 CrossRef Medline

16. Orphanidou-Vlachou E, Auer D, Brundler MA, et al. (1)H magnetic resonance spectroscopy in the diagnosis of paediatric low grade brain tumours. Eur J Radiol 2013;82:e295-301 CrossRef Medline

17. Quon $\mathrm{H}$, Brunet $\mathrm{B}$, Alexander A, et al. Changes in serial magnetic resonance spectroscopy predict outcome in high-grade glioma dur- ing and after postoperative radiotherapy. Anticancer Res 2011;31: 3559-65 Medline

18. Clinical Trial Imaging Endpoint Process Standards Guidance for Industry (Draft Guidance March 2015). http://www.fda.gov/ucm/ groups/fdagov-public/@fdagov-drugs-gen/documents/document/ ucm268555.pdf. Accessed October 19, 2015

19. Ellingson BM, Bendszus M, Boxerman J, et al; Jumpstarting Brain Tumor Drug Development Coalition Imaging Standardization Steering Committee. Consensus recommendations for a standardized brain tumor imaging protocol in clinical trials. Neuro Oncol 2015; 17:1188-98 CrossRef Medline

20. Ellingson BM, Kim HJ, Woodworth DC, et al. Recurrent glioblastoma treated with bevacizumab: contrast-enhanced T1-weighted subtraction maps improve tumor delineation and aid prediction of survival in a multicenter clinical trial. Radiology 2014;271:200-10 CrossRef Medline 